\title{
Das Land, wo die Laien Dialektexperten sind
}

Zusammenfassung: In der Deutschschweiz wird Sprache häufig zum alltäglichen Gesprächsgegenstand gemacht. Dies ist u. a. der polydialektalen Konstellation geschuldet, die die Schweizer Sprachsituation auszeichnet: Schweizerinnen und Schweizer kommen in ihrem Alltag laufend mit unterschiedlichen Sprachen und Dialekten in Kontakt. Dieser Kontakt generiert laienlinguistisches Wissen, das individuell je unterschiedlich ausgestaltet sein kann, insgesamt aber als verhältnismässig differenziertes Wissen gelten kann. Vorliegender Beitrag versucht, den Stellenwert des dialektalen Alltagswissens in der Schweiz und dessen Differenziertheit über zwei Zugänge nachzuzeichnen: Einerseits werden 45 Sendungen der Schweizer Radio-Talksendung Persönlich, die im Jahr 2019 ausgestrahlt wurden, auf spontane Dialektthematisierungen hin besehen: Wie oft wird der Dialekt - der nicht im Zentrum der Sendung steht - dort zum Gesprächsthema gemacht? Nach welchen inhaltsbezogenen Aspekten lassen sich die Dialektthematisierungen ordnen? Andererseits werden Dialektthematisierungen diskutiert, die im Rahmen des Forschungsprojekts Ländere ${ }^{n}$ erhoben wurden und damit als elizitiert gelten können. Ein Teil dieser Dialektthematisierungen gibt Aufschluss über repräsentierte laienlinguistische Konzepte, ein anderer über repräsentierte laienlinguistische Perzepte. Die Kontrastierung dieser beiden Wissenstypen erlaubt detaillierte Rückschlüsse über die Granularität und die Zugänglichkeit laienlinguistischen Wissens.

Abstract: In German speaking Switzerland people often talk about language in everyday conversations. This is in part due to the polydialectal constellation, which characterizes the Swiss language situation: In their everyday lives, Swiss are continually in contact with different languages and dialects. This contact generates linguistic knowledge in laypersons, which may vary from one individual to another, but as a whole can be considered to be quite differentiated. This paper attempts to show the significance of dialectical everyday knowledge in Switzerland and its degree of differentiation by applying two approaches. On the one hand, 45 episodes of the Swiss radio talk show Persönlich that were

Christen, Helen: Universität Freiburg, Germanistische Linguistik, Av. de l’Europe 20, 1700 Freiburg, helen.christen@unifr.ch

Schiesser, Alexandra: Pädagogische Hochschule Zug, Zentrum Mündlichkeit, Zugerbergstrasse 3, 3006 Zug, alexandra.schiesser@phzg.ch 
broadcast in 2019 are examined for spontaneous thematizations of dialects: How often is the dialect - which is not the focus of the program - made the subject of conversation? According to which contents can the thematizations of dialect be classified? On the other hand, statements about dialect are discussed which were collected in the context of the research project Ländere ${ }^{n}$ and can thus be considered as elicited. Part of these dialect thematizations provide information about the way laypersons represent linguistic concepts, others about the laypersons' respondent reactions to actual linguistic data. The contrast between these two types of knowledge allows detailed conclusions on the granularity and accessibility of laypersons' linguistic knowledge.

Schlüsselwörter: Deutschschweizer Sprachsituation, laienlinguistische Metakommunikation, polydialektale Konstellation, sprachliches Alltagswissen, Wahrnehmungsdialektologie, language situation in German-speaking Switzerland, linguistic metacommunication of laypersons, polydialectal constellation, everyday linguistic knowledge, perceptual dialectology

\section{Einführung}

Der Moderator der Talksendung Persönlich, die wöchentlich am Sonntagmorgen im Radio SRF 1 ausgestrahlt wird ${ }^{1}$ und jeweils zwei Personen ans Mikrophon holt, stellt einen der beiden Gäste wie folgt vor:

\section{Beispiel (1) (31.03.2019)}



\footnotetext{
1 Im Sendungsportrait ist zu lesen: „,Persönlich“ ist kein heisser Stuhl und auch keine Informationssendung, sondern ein Gespräch zur Person und über ihr Leben. Jeden Sonntag sprechen Menschen über ihre Wünsche, Interesse, Ansichten und Meinungen." Vgl. https://www.srf.ch/sendungen/persoenlich (letzter Zugriff 23.02.2021); unter dieser Adresse ist ebenfalls das Archiv der Sendungen zugänglich, das für die Ausführungen in Abschnitt 2 genutzt wird.
} 
Standarddeutsche Übertragung: ,Dass er ursprünglich aus dem Thurgau kommt, das hört man seinem Dialekt noch immer an. Der zweiundsechzigjährige Christoph Vögele.

Es ist in der Deutschschweiz keineswegs ungewöhnlich, dass Sprache zum alltäglichen Gesprächsgegenstand wird und der Dialekt eines Sprechers, einer Sprecherin - wie in Beispiel (1) ersichtlich - gar vorrangig thematisiert und in Bezug zu sprachräumlichen Aspekten einer individuellen Biographie gebracht wird: Nicht der Sachverhalt des Dialektsprechens an sich ist dabei für den Moderator bemerkenswert, vielmehr ist die Sprache des Gastes ein willkommener Einstieg, um dessen räumliche Herkunft zu thematisieren. Der Idiolekt des Gastes scheint aus der Sicht des Moderators nicht zum Ausstrahlungsort der Radiosendung - Solothurn - zu passen, sondern offenbar eine ostschweizerische Herkunft anzuzeigen.

Die sozialen Konventionen des Sprach(formen)gebrauchs in der Deutschschweiz, der sich - je nach theoretischem Zugriff - als Diglossie oder Bilingualismus modellieren lässt (vgl. Schmidlin \& Franceschini 2019), sehen für die Mündlichkeit den Dialekt als unmarkierte Varietät vor. Da es kein Schweizerdeutsch als eine Art von Koine gibt, die über den einzelnen Dialekten stünde, entstehen polydialektale Konstellationen (zum polydialektalen Dialog vgl. Ammon 1995: 294). Dass diese kaum kommunikationshinderlich sind, verdankt sich - nebst dafür unabdingbaren strukturellen Ähnlichkeiten der alemannischen Dialekte - der Voraussetzung, dass der Dialekt sozial erwünscht ist und man sich nicht nur auf dialektale Variation einlässt, sondern diese nachgerade erwartet. Die rezeptiven Routinen, die sich aus dem anhaltenden Umgang mit dialektaler Variation ergeben, rüsten die Deutschschweizer/innen mit einer „passiven Polydialektalität“ (Glaser 2014: 52) aus, wobei diese gleichermassen als Voraussetzung und als Effekt eines Jede/r-spricht-seinen/ihren-DialektPrinzips gelten kann. ${ }^{2}$

2 Die Erfahrung der Polydialektalität gehört zum (heutigen) Alltag eines jeden Deutschschweizers, einer jeden Deutschschweizerin, wobei deren zunehmende Mobilität und deren Medienkonsum dazu einen wesentlichen Beitrag leisten. So sind etwa nach Ausweis des Bundesamtes für Statistik von 4 Mio. Arbeitspendlern im Jahre $201751 \%$ solche, die innerhalb von verschiedenen Gemeinden ihres Wohnkantons pendeln und $20 \%$ solche, deren Arbeits- und Wohnort in zwei verschiedenen Kantonen liegt. Der zurückgelegte durchschnittliche Arbeitsweg beträgt $15.0 \mathrm{~km}$ und stellt einen Zuwachs von $16 \%$ gegenüber dem Jahr 2000 dar, vgl. https://www.bfs.admin.ch/bfs/de/home/statistiken/mobilitaet-verkehr/personenverkehr/ pendlermobilitaet.html (letzter Zugriff 23.02.2021). 
Die dialektalen Idiolekte, die in Deutschschweizer Konstellationen ins Spiel kommen, sind zwangsläufig indexikalisch, weil sich dialektale Varianten gerade dadurch auszeichnen, mit Räumen verknüpft zu werden: Die sprachlichen Eigenheiten von Ortsansässigen werden zu Raumindikatoren gemacht, so dass - umgekehrt - diese Eigenheiten dann grössere oder kleinere Gebiete der Deutschschweiz abzurufen vermögen, und dies gänzlich unabhängig davon, welchen tatsächlichen Bezug ein Sprecherindividuum zu diesen Räumen hat.

Dieses angesprochene Sprache/Raum-Wissen setzt nicht zwingend einen persönlichen Kontakt mit Sprecherinnen und Sprechern unterschiedlicher Dialekte voraus, sondern dieses kann auch durch Tradierung weitergegeben werden und sowohl „positives“ wie „negatives Varietätenwissen“ (Hundt 2017: 124-138) umfassen. Während das positive Varietätenwissen eine unterschiedliche Granularität (von „unspezifischer Ahnung“ bis zu spezifischem Wissen von „Einzelmerkmalen“) haben kann, meint das negative Varietätenwissen das Fehlen von Konzeptualisierungen. Dazu reiht Hundt (2017: 125-126) auch einen Wissenstyp, „bei dem die GPn zwar auch keine näheren Angaben zu den Sprechweisen oder deren Sprecher machen können, bei denen sie aber immerhin über Bezeichnungen für die Dialektregion verfügen.“ Der Feststellung, wonach man hier nicht von „gänzlichem Unwissen“ (Hundt 2017: 126) ausgehen kann, ist beizupflichten, da diese Bezeichnungen Indizien dafür sind, dass über Analogieschlüsse - von gebietsspezifischem Sprachgebrauch ausgegangen wird. Zudem lassen Dialektbezeichnungen selbst ein sprachräumliches Organisationsprinzip erkennen (zu Wissensorganisation nach schweizerischen Kantonen vgl. Stoeckle \& Schwarz 2019).

Die angesprochenen - in ihrer Ausprägung sehr heterogenen - sprachbezogenen Wissensbestände werden als Teil des Alltagswissens betrachtet. Dieses wird - nach Hundt (2017: 138-139) - sozialkonstruktivistisch

als ein Wissen verstanden, das nicht fachspezifisch ist (kein Expertenwissen), das potentiell allen Sprachteilnehmern zur Verfügung steht, das sowohl prozedurale als auch deklarative Bestandteile enthält, das nur teilweise von den Laien explizierbar ist, das jedoch wichtige Funktionen in der Bewältigung des Alltags übernehmen kann.

Mit Alltagswissen, dem ein „Sonderwissen“ (Schütz \& Luckmann 2003: 418) gegenübersteht, ist die gesellschaftliche Verteilung des Wissens angesprochen, von Wichter (1995) als „Vertikalität des Wissens“ bezeichnet, dem unterschiedliche Wissensträger entsprechen. Schütz \& Luckmann (2003: 443) unterscheiden dabei zwischen „Laien“, „Gutinformierten“ und „Sachverständigen“, während Hoffmeister (2019: 166) - nach Massgabe des Professionalisierungsgrades - ein Kontinuum mit den Wissensträgern „Laie“, „Amateur“, „Experte“ und 
„Profi“ aufspannt. Das unterschiedliche Wissen ergibt sich aus unterschiedlichen Haltungen und Interessen gegenüber der Welt, die mit einer unterschiedlichen Bereitschaft einhergehen, Gegebenheiten als selbstverständlich resp. nicht selbstverständlich anzunehmen. So zeichnet sich das Alltagswissen von Laien dadurch aus, dass es weder hinterfragt wird noch hinterfragt zu werden braucht, weil es trotz Lückenhaftigkeit und Oberflächlichkeit ausreichend ist, um den Alltag erfolgreich zu bewältigen. Das Sonderwissen von „Sachverständigen“ oder „Profis“ dagegen betrifft einen schmalen Ausschnitt der fassbaren Welt und ist „an dem Ideal ausgerichtet, konsistent und begründet zu sein“ (Schnettler 2007: 110).

Die Interessen selbst sind von einem Relevanzsystem geleitet, das darüber entscheidet, welches Ausmass an Explizitheit einem Wissensbereich angemessen ist und wo die ,Selbstverständlichkeiten' des Alltagswissens aufgebrochen werden sollen. Alltagswissen und Sonderwissen sind damit nicht voneinander getrennt, sondern hierarchisch miteinander verzahnt:

Alle Begriffe und Modelle der Wissenschaften (,Konstrukte zweiter Ordnung') lagern auf den Strukturen von Alltagskonstrukten (,erster Ordnung') auf. Anders als diese müssen jene andererseits - den spezifischen Relevanzsetzungen der Wissenschaft folgend - dem Postulat ,logischer Konsistenz‘ genügen.

(Schnettler 2007: 109)

Dass der Bereich ,Sprache/Dialekt` in der Deutschschweiz auch von ,NichtProfis' nicht bloss thematisiert, sondern mit einem gewissen Detailwissen verhandelt wird, ist nicht dem Gegenstandsbereich an sich geschuldet, sondern hängt von dessen gesellschaftlicher Relevanz „für das Selbstverständnis und das Traditionsbewußtsein“ (Schütz \& Luckmann 2003: 401) der Deutschschweizerinnen und Deutschschweizer ab (zur Bedeutung des Dialekts für das Deutschschweizer Selbstverständnis vgl. z. B. Trümpy 1955; Schwarzenbach 1969; Ruoss \& Schröter 2020). Es ist also zumindest hypothetisch davon auszugehen, dass das sprach-/dialektbezogene Alltagswissen in unterschiedlichen Regionen des deutschsprachigen Raumes in Abhängigkeit von gesellschaftlichen Relevanzen ungleich ausgebildet ist.

Im Hinblick auf die Rekonstruktion des sprachlichen Alltagswissens werden nachfolgend zwei Datentypen auf Metakommunikate hin besehen: In Radiosendungen wird die (relativ spontane und unvorhersehbare) Thematisierung von Sprachlichem in den Blick genommen (Abschnitt 2); aus einer Datenerhe- 
bung im Rahmen eines grösseren Forschungsprojektes ${ }^{3}$ werden die Befragungen zu Dialekten, ihren Arealen und ihren Merkmalen ausgewertet (Abschnitt 3).

\section{Metadialektales in polydialektaler Konstellation}

Die eingangs erwähnte Radiosendung Persönlich wird hier nochmals in den Blick genommen, und zwar dienen die 45 Sendungen, die im Jahr 2019 ausgestrahlt wurden ${ }^{4}$, dazu, um einerseits Anhaltspunkte darüber zu erhalten, wie oft Sprachliches in einem Kontext verhandelt wird, der nicht auf Metakommunikation hin angelegt ist ${ }^{5}$, und andererseits, um die inhaltlichen Dimensionen sprachlichen Alltagswissens rekonstruieren zu können.

In fünfzehn, d. h. einem Drittel der Sendungen wird Sprachliches zum Thema, sei es von Seiten der Moderation (vgl. Beispiele 1 und 7) oder von Seiten der Eingeladenen. Die Metakommunikate lassen sich induktiv nach den in-

3 Forschungsprojekt „Ländere ${ }^{n}$ - Die Urschweiz als Sprach(wissens)raum“; Laufzeit: 20122017; unterstützt vom Schweizerischen Nationalfonds. In diesem Projekt wurden subjektive und objektive Sprachdaten von 60 Probandinnen und Probanden an vier Orten im Kanton Obwalden (Sarnen, Lungern, Melchtal, Engelberg), drei Orten im Kanton Nidwalden (Stans, Hergiswil, Emmetten) und einem Ort im Kanton Uri (Seelisberg) erhoben; vgl. http://p3.snf.ch/project-140287 (letzter Zugriff 23.02.2021).

4 Über die Sommermonate hinweg werden keine neuen Live-Sendungen gemacht, sondern ältere Sendungen wiederholt.

5 Es ist im Blick zu behalten, dass diese Talksendung jeweils von einem Moderator (mit Solothurner oder Basler Dialekt) oder einer Moderatorin (mit Zürcher oder Berner Dialekt) geleitet wird. Persönlich wird aus unterschiedlichen Deutschschweizer Orten gesendet, wo bevorzugt öffentliche Räume für die Veranstaltung genutzt werden. Neben den zwei Gästen ist am Veranstaltungsort immer auch Publikum anwesend, das zwar kein Rederecht hat, sich aber etwa durch Lachen oder Applaus bemerkbar macht. Die Eingeladenen haben zumeist, aber nicht zwingend, einen Bezug zum jeweiligen Sendeort. Verstehen die Eingeladenen schweizerdeutschen Dialekt, ist die Sprachform der Moderatoren Schweizerdeutsch - so auch in der Sendung vom 06.01.2019, wo ein bundesdeutsch-schweizerisches Ehepaar (mit Berliner Wohnsitz) zu Gast ist. Die Ehefrau mit deutschem Hintergrund (und Dialektverstehenskompetenz) spricht Hochdeutsch, der Moderator und der Ehemann sprechen Dialekt, ausser letzterer wendet sich direkt an seine Frau. Je nach Moderationsperson und je nach Gästen ergibt sich ein polydialektaler Dialog, in den unterschiedliche dialektale Varietäten involviert sind (vgl. Christen 2008). Die Gesprächsthemen sind vorgängig nicht abgesprochen, kreisen aber vorhersehbar um die Themen Lebenslauf, Beruf, Familie und Freizeit (vgl. Fussnote 1). 
haltsbezogenen Aspekten ,Sprachbiographisches‘ (Welche Erfahrungen hat ein Individuum mit seiner eigenen Varietät, seinem eigenen Varietätenrepertoire gemacht?), ,Verständlichkeit‘ (Ist eine dialektale Variante, eine dialektale Varietät verständlich?), ,Evaluation“ (Welche Bewertungen zieht eine dialektale Variante, eine dialektale Varietät auf sich?) und ,Raum“ (Wohin und wem ,gehört' eine dialektale Variante, eine dialektale Varietät?) ordnen, wobei diese verschiedenen Aspekte teilweise miteinander verknüpft werden. ${ }^{6}$

Dem Sendekonzept geschuldet, steht (Sprach-)Biographisches im Vordergrund, das - obwohl auf individuellen Erfahrungen beruhend - über allgemeines Alltagswissen konzeptualisiert wird. Häufig wird das sprachliche Repertoire der Gäste Gesprächsgegenstand, die - über mehrere Varietäten verfügend öfters auch deren Gebrauch thematisieren:

\section{Beispiel (2) (08.09.2019)}

(1) $\{8: 00\} \mathrm{RF}: \quad<<$ rall> i bö met zWÖÖ spraache ufgwachse>,

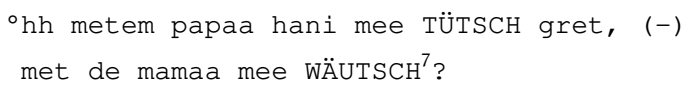

,Ich bin mit zwei Sprachen aufgewachsen, mit dem Papa habe ich mehr Deutsch geredet, mit der Mama mehr Französisch.“

Im Zusammenhang mit Varietätenrepertoires wird ebenso zum Gesprächsgegenstand, wie Varietäten erworben werden, wobei Kindern nachgesagt wird, dass diese problemlos ihre Umgebungssprache(n) erwerben, wie dies eine Eingeladene der Talksendung von ihrer Tochter erzählt, die sich beim Umzug von Kiel nach Solothurn problemlos eine neue Varietät angeeignet habe:

\section{Beispiel (3) (27.10.2019)}

(1) $\{25: 23\} \quad B G$ : ennerhalb vomene haube jaar het si <<rall> soloTOORnerisch gleert $>$ ? das esch KLAAR=

,Innerhalb eines halben Jahres hat sie Solothurnerisch gelernt. Das ist klar.‘

Ein Gast aus einer italophonen Familie berichtet dagegen, dass er seine zweite Varietät nicht in optimaler Qualität erworben habe:

6 Tophinke \& Ziegler (2014: 226-232) können in spontaner Dialektthematisierung in der Weblogkommunikation folgende semantische Topoi festmachen: „Reinheits- und Echtheitstopos“, „Topos der Normativität“, „Verständlichkeitstopos“, „Topos von der Schönheit der Dialekte“ und „Angemessenheitstopos“.

7 welsch i. d. B. ,französisch'. 


\section{Beispiel (4) (24.02.2019)}

(1) \{31:10\} CR: $\begin{aligned} & \text { aso } i \text { ha DENN (.) na ned wörkli dütsch } \\ & \text { chönne, }= \\ & =i \text { ha daas wörkli deet im chendergaarte } \\ & \text { gleert (1.5) ((räuspert sich)) (1.5) } \\ & \text { ond miis bäärndütsch isch } \circ \text { höt so (2.0) } \\ & <<t>\text { mettu> }\end{aligned}$

,Also ich habe dann noch nicht wirklich Deutsch gekonnt. Ich habe das wirklich dort im Kindergarten gelernt. Und mein Berndeutsch ist auch heute so mittel.

An eine temporäre Kompetenz-Asymmetrie zwischen ihren Varietäten will sich eine Gesprächsteilnehmerin erinnern, die als Kind von Japan in die Schweiz gezogen ist:

\section{Beispiel (5) (21.04.2019)}

$$
\{37: 18\} \quad \text { CS: }
$$

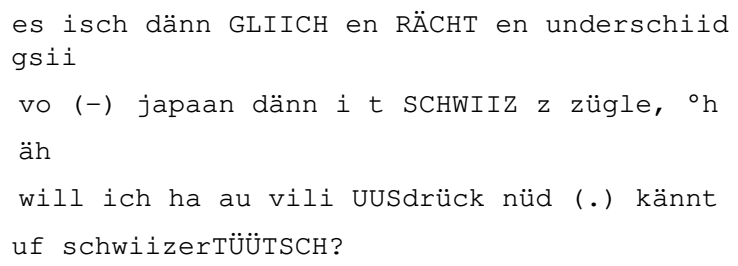

,Es ist dann doch ein rechter Unterschied gewesen. Von Japan dann in die Schweiz umzuziehen, weil ich habe auch viele Ausdrücke nicht gekannt auf Schweizerdeutsch.

Ein anderer Gast erwähnt ein einzelnes Merkmal, welches er als letzte Hürde überwunden habe, um den Dialekt der neuen Lebensumgebung so zu sprechen, dass er nicht länger als Fremder ${ }^{8}$ wahrgenommen worden sei:

\section{Beispiel (6) (12.05.2019)}

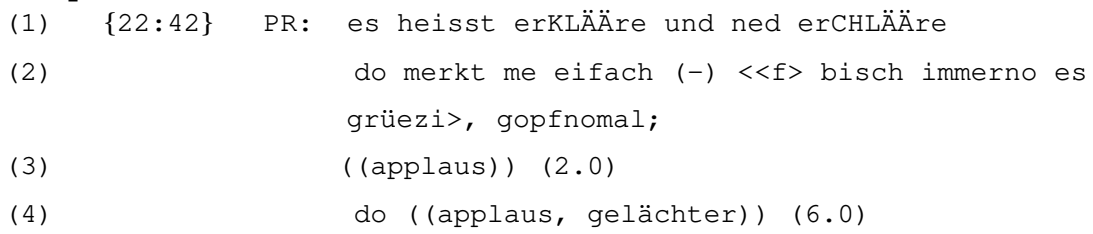

8 Als „Grüezi“ (n.) werden im Wallis scherzhaft jene bezeichnet, die als Gruss „Grüezi“ gebrauchen und sich damit als Nicht-Walliser zu erkennen geben. 
(5)

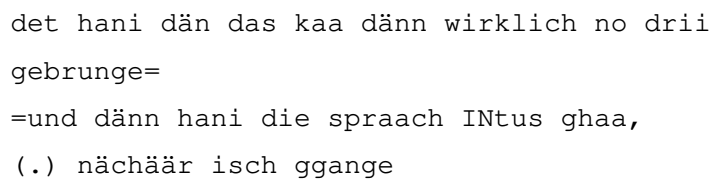

,Es heisst «erklääre» und nicht «erchlääre». Da merkt man einfach: Du bist immer noch ein «Grüezi» (Nicht-Walliser), verflixt, dort habe ich dann dieses «K» dann wirklich noch dreingebracht, und dann habe ich dieses Sprache intus gehabt. Nachher ist es gegangen.

Es sind nun ausnahmslos die Moderatoren der Sendung, die das Thema ,Verständlichkeit‘ initiieren und ihre Gäste um Beispiele bitten, die die - auf diese Weise suggerierten - Verstehensschwierigkeiten in einem neuen Sprach-/Dialektgebiet illustrieren sollen:

\section{Beispiel (7) (14.04.2019)}

(1) $\{14: 19\} \quad M$ : äh_SI händ ja (-) dä dialäkt au müesse LEEre

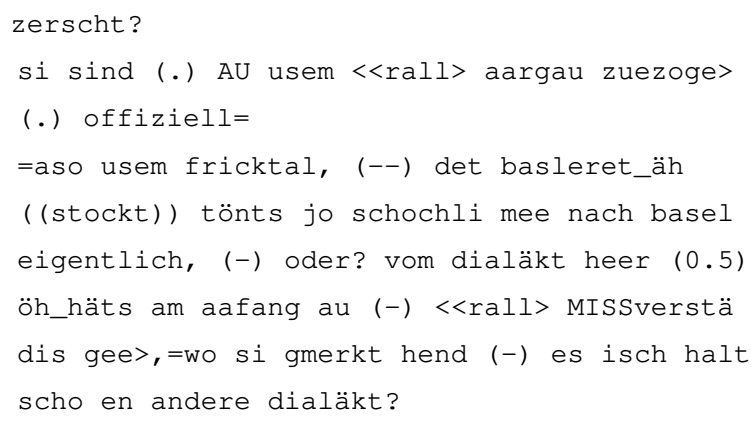

,Sie haben ja diesen Dialekt auch zuerst lernen müssen. Sie sind auch aus dem Aargau zugezogen, offiziell also aus dem Fricktal. Dort «baslern»... klingt’s ja schon ein wenig mehr nach Basel eigentlich, oder, vom Dialekt her? Hat es am Anfang auch Missverständnisse gegeben, bei denen Sie germerkt haben, es ist halt schon ein anderer Dialekt?`

Bereitwillig geben die Gäste Anekdotisches zum besten, das naturgemäss (poly-)lexikalische Grössen betrifft, die für Verwirrung sorgen sollen: Die heteronymen Bezeichnungen einer traditionellen Schweizer Wurst - Cervelats oder Klöpfer - führen anfänglich zu Missverständnissen beim Metzger (17.04.2019); acht Tage in der Bedeutung, eine Woche‘ sorgt beim Kind, das gelernt hat, dass die Woche sieben Tage hat, für Erstaunen (17.04.2019); der Coiffeur wird in der neuen Lebensumgebung (wohl humoristisch, aber zunächst unverständlich) als Höörlidieb ,Härchendieb“ bezeichnet (21.04.2019). Die relative Harmlosigkeit der 
Beispiele lässt jedoch erkennen, dass die Talkgäste kaum je mit wirklich ernsthaften interdialektalen Verstehensproblemen konfrontiert waren oder sind, dass sie aber in der Lage sind, sprachliche Variation wahrzunehmen, diese - zu Recht oder zu Unrecht - als dialektal zu kategorisieren, und - als immer wieder erzählte Anekdoten - gleichzeitig den Mythos fortschreiben, wonach die Deutschschweizer Dialekte derart verschieden seien, dass es zu Missverständnissen komme. ${ }^{9}$ Der Aspekt ,Verständlichkeit' provoziert im Persönlich-Format am meisten Nennungen sprachlicher (meist lexikalischer) Merkmale; diese feinkörnige Wissensschicht verdankt sich aber dem Insistieren der Moderationspersonen auf ,Belegen‘.

Die evaluativen Metakommunikate beziehen sich auf verschiedene sprachliche Ebenen, so beispielsweise auf den Sachverhalt eines Varietätenrepertoires. Besteht das individuelle Repertoire aus mehreren Varietäten, wird dies in den Talksendungen als Bereicherung dargestellt (,scho e groossi beriicherig“, 23.06.2019) oder als im Dienste erwünschter Kulturvermittlung gesehen (,ich uusschliesslich schwiizertütsch gret ha mit im [in englischsprachigem Umfeld, H. C. \& A. S.], um die kultuur die schwiizerkultuur im z zeige“, 27.10.2019). Evaluative Metakommunikate können auch Varietäten selbst betreffen, wobei in den Sendungen ausnahmslos der Dialekt (im Vergleich zu einer anderen Sprache) oder bestimmte dialektale Ausprägungen bewertet werden. So entdeckt eine Sängerin, die früher vornehmlich französische Chansons gesungen hat, für ihre Liedtexte neuerdings den Dialekt, den sie im Vergleich zum Französischen für „harzig, kratzig“, aber gleichzeitig auch für „melodiöser, weiblicher, feiner“ (usw.) hält (08.09.2019). ${ }^{10}$ In einer anderen Gesprächsrunde sind sich zwei Basler darin einig, dass der eine Gast in seinem Beruf als Schauspieler - wegen seines Dialekts - zwangsläufig Bösewichte spielen müsse und als Sprecher für Produktewerbung ungeeignet sei. ${ }^{11}$ Schliesslich berichtet ein Talkgast davon, in

$9 \mathrm{Zu}$ interdialektalen Verstehbarkeitsproblemen in der Talkshow Persönlich, vgl. Christen (2008).

10 Widersprüchlichkeiten sind bei der Äusserung von Meinungen weder ungewöhnlich noch stossend, da der Anspruch an Konsistenz bei Alltagswissen - im Gegensatz zum wissenschaftlichen Sonderwissen - vergleichsweise gering ist (zu „Eigenschaften und Funktionen von Alltagswissen“vgl. Hundt 2017: 138-146).

11 Die aktuellste Befragung zum Gefallen von Dialekt ist vom Customer-Insight-Institut der Universität St. Gallen 2019 durchgeführt worden, dessen Leiter A. Herrmann an dieser Stelle für die Einsicht in die Ergebnisse gedankt sei. Mittels einer repräsentativen Umfrage mit 3750 Befragten wurde das Image verschiedener Schweizer Städte ermittelt. Unter den gegen zwanzig Themen, die als das Image mutmasslich beeinflussende Faktoren abgefragt wurden, fungierte auch die Beliebtheit des Dialekts. Die Zustimmung zum Satz „Sie empfinden den gesprochenen 
einer ärmeren Gegend mit vielen Migranten aufgewachsen zu sein, und dort „nicht mehr richtig Schweizerdeutsch“ gesprochen zu haben. Sie habe damals aufgehört, die „Wörter zu konjugieren und zu deklinieren“. Dieses „Jugodütsch“ habe sich dann später wieder ,ausgewachsen“ (03.02.2019).

Was den Zusammenhang von Sprache und Raum betrifft, so zeigt sich in mehreren Bekundungen, dass bloss einem Ausschnitt aus dem örtlichen Variationsspektrum der Status des eigentlichen, richtigen Ortsdialekts zugewiesen wird. Der Ortsdialekt wird an Autochthonen festgemacht. Wie Beispiel (4) zeigt, wird die fluide soziale Kategorie der Autochthonie dabei relativ streng ausgelegt: Ein Berner mit Migrationshintergrund hält sein Berndeutsch, das er im Kindesalter erworben hat, - kleinlaut formuliert - für bloss „mittel“. Ebenso glaubt eine Frau, die im Alter von zehn Jahren nach Schaffhausen gekommen ist, keine kompetente Sprecherin des Schaffhauserdeutschen zu sein:

\section{Beispiel (8) (14.04.2019)}

(1) $\{10: 23\}$ UB: ICH chas bes höt ned?, (---) aso $t$ schaff [huuser-]



,UB : Ich kanns bis heute nicht, also die Schaffhauser ... M : doch das... UB : nein, nein also, mein Mann ist Schaffhauser und ich spreche also nicht wie er. Und das rollende «R», das werde ich ich auch irgendwie nicht ganz los.

In diesen alltäglichen Metakommunikaten manifestiert sich ein „Sedentarismus“, wie er gleichermassen für die traditionelle dialektologische Sprache/Raum-Konzeptualisierung typisch ist, bei der das wissenschaftliche Konstrukt Basisdialekt/Grundmundart über ,immobile' Sprecher operationalisiert und der Sprachgebrauch ,mobiler` Sprecher als dialektologischer Forschungs-

Dialekt als sehr angenehm.“ (1: stimme überhaupt nicht zu 7: stimme voll und ganz zu) sollte dabei in Bezug auf verschiedene Städte skaliert werden. Gemäss Prestons (2010: 24) Taxonomie der Techniken der perceptual dialectology handelt es sich dabei um eine Abfrage mit der Charakteristik „,internal \& conscious“ (Preston 2010: 6), bei der offen Bewertungen von ,Konzepten in den Köpfen“ der Befragten eingefordert werden. 
gegenstand ausgeblendet wird (vgl. Britain 2016). Aus dem Sprachverhalten als autochthon geltender Sprecher wird also sowohl das dialektologische Alltagsals auch das Fachwissen konstituiert. ${ }^{12}$ Während jedoch das Fachwissen deskriptiver Natur ist, hat das Alltagswissen deutlich normative Komponenten: Der Dialekt Autochthoner gilt als ,besser' als andere Ausprägungen. Die TalkTeilnehmerin, die sich die Kompetenz des Schaffhauserdeutschen abspricht (Beispiel 8), gleicht ihren Idiolekt ab mit jenem ihres ,ausreichend‘ autochthonen Mannes, den sie als „Schaffhauser“ bezeichnet. Die Unterschiede gelten ihr dann alltagslogisch als Evidenzen für ihre mangelnde (dialektale) Ortszugehörigkeit. Diese sieht sie auch bestätigt in ihrer „rollenden“/r/-Realisierung, die nicht dem Ostschweizer Schibboleth eines „nicht-rollenden“ /r/ entspricht. Der Sprachatlas der deutschen Schweiz (SDS II: Karten 151-153) weist basisdialektal für den Ortspunkt Schaffhausen SH 7 allerdings sowohl rollendes als auch nicht-rollendes /r/ aus. Es scheint, dass die sich als „Nicht-Schaffhauserin“ Einschätzende ihren Fokus auf die Abweichungen zu ihrem Idiolekt legt und die Gemeinsamkeiten ausblendet, um ihr Selbstbild als einer Nicht-Autochthonen zu bestätigen resp. um Kongruenz herzustellen zum Alltagswissen, wonach nur ,wirklich' Einheimische den ,richtigen' Dialekt sprechen würden.

Die Sprache/Raum-Verknüpfung kann nun in Alltagsvorstellungen dergestalt essentialisiert werden, dass ,exterritoriale‘ Sprecher eines Dialekts negative Sanktionen zu gewärtigen haben. Eine aus dem Kanton Graubünden stammende Frau, die in ihrem Elternhaus mit Rätoromanisch und Berndeutsch aufgewachsen ist, darf bei ihren Medienauftritten nicht Berndeutsch sprechen, sondern sie muss - um der Erwartung des Publikums gerecht zu werden - als Bündnerin Bündnerdeutsch sprechen:

\section{Beispiel (9) (03.11.2019)}

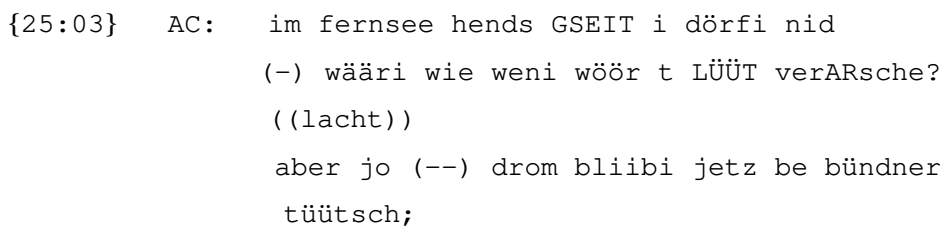

12 Während die Autochthonizität in der Wissenschaft über Sprechermerkmale klar definiert ist, ist diese im Alltag eine wohl eher prototypisch organisierte Kategorie. Gemeinsam ist aber der wissenschaftlichen und der Alltagskonzeption, dass sie gleichermassen auf einer sedentaristisch ausgerichteten Dialektideologie fussen, die in der Zeit der Romantik wurzelt (vgl. Bucholtz 2003). 
,Beim Fernsehen haben sie gesagt, ich dürfe nicht. (Es) wäre, als wenn ich die Leute verarschen würde. Aber ja, darum bleibe ich jetzt bei Bündnerdeutsch.

Mit dem Gebrauch eines Berner Dialekts würde sie eine ,falsche‘ lokale Fährte legen, inszenierte sie sich doch sprachlich als zum Ort Bern zugehörig, was ihr aber als ,Exterritorialer' nicht zusteht. Umgekehrt ist es legitim, wenn man in einer neuen Lebensumgebung an seinem ,mitgebrachten“ Dialekt erkennen lässt, woher man ,eigentlich' kommt (vgl. Beispiel 1). Will man allerdings ,dazu“ gehören, kann aus der Warte des Sprechers in Beispiel (6) schon ein einzelnes Merkmal ausschlaggebend dafür sein, ob man als aussenstehendes „Grüezi“ (i. S. v. ,Nicht-Walliser', vgl. Fussnote 7) betrachtet wird oder nicht. ${ }^{13}$

Der Anlage der Radiosendung geschuldet, stehen die Metakommunikate im Dienste einer differenzierten Portraitierung der Talkgäste. Mit der Thematisierung von Varietätenrepertoires und deren Erwerb, von Dialekten, die man mehr oder weniger , gut ${ }^{*}$ - spricht, bringen Gäste und Moderatoren Facetten ins Spiel, die räumliche Mobilität und soziale (Nicht-)Zugehörigkeiten in den Fokus rücken. Dafür sind in vielen Fällen grobkörnige allgemeine Dialektcharakterisierungen ausreichend, während einige Gäste es verstehen, zur allfälligen Stützung ihrer Argumentation durchaus auch feinkörniges spezifisches Dialektwissen geltend machen (vgl. Beispiele 6 und 8).

\section{Metadialektales in linguistischen Befragungen}

Während im vorangehenden Abschnitt Metakommunikate untersucht wurden, die - bezogen auf den kommunikativen Kontext, in dem sie geäussert wurden als relativ spontan und unvorhersehbar eingeschätzt werden können, handelt es sich bei den nachfolgend präsentierten Metakommunikaten um einen gänzlich anderen Datentyp. Diese Metakommunikate wurden im Kontext des Forschungsprojekts Ländere ${ }^{n}$ in der Innerschweiz elizitiert (vgl. Fussnote 3); sie haben demnach einen vergleichsweise geplanten resp. extrinsisch motivierten Charakter. ${ }^{14}$ In Abschnitt 3.1 werden zunächst Metakommunikate präsentiert,

13 Das Beispiel (6) liest sich quasi als aktuelle (und nicht-martialische) Version der Passage

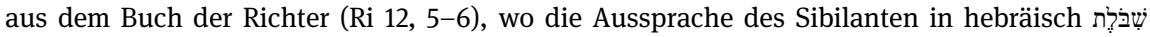
(,Strömung, Flut“ oder ,Ähre`) als „Schibboleth“ oder „Sibboleth“ die (folgenreiche) Zugehörigkeit zu einer Volksgruppe offenlegte.

14 Dies soll keineswegs heissen, dass die Metakommunikate den Probandinnen und Probanden bei der Erhebung in den Mund gelegt wurden: Methodisch wurde bei der Befragung äus- 
die mit Preston Rückschlüsse auf laienlinguistische ,Konzepte“ geben, die die Eigenschaft von „inner resources“ (Preston 2010: 1) haben. Diese (rekonstruierten) Konzepte entstammen sogenannten Draw-a-map-Tasks, in denen die Probandinnen und Probanden gebeten wurden, auf zwei Karten je unterschiedlichen regionalen Zuschnitts und unterschiedlicher Informationsdichte ihnen bekannte Dialektgebiete einzuzeichnen, sie zu benennen und Überlegungen dazu anzustellen, was sie - sprachlich wie nichtsprachlich - mit diesen Gebieten verbinden. In Abschnitt 3.2 stehen Metakommunikate im Fokus, die mit Preston (2010: 2) als „respondent reactions to actual linguistic data“ und damit als ,Perzepte‘ eingestuft werden können. Sie wurden aus sogenannten ,Hörproben-Aufgaben' gewonnen, bei denen die Probandinnen und Probanden acht Sprechproben aus unterschiedlichen Schweizer Regionen verorten und ihre Verortung an sprachlichen Merkmalen festmachen mussten. In beiden Abschnitten interessieren nachfolgend Probandinnen und Probanden, die vergleichsweise viel oder wenig metasprachliches Wissen mit unterschiedlicher Granularität verbalisieren. Dieser Zugriff soll Aufschluss darüber geben, welches Wissensspektrum bei den linguistischen Laien der vorliegenden Stichprobe vorhanden ist und ob das Wissensspektrum abhängig von der Methodik der Erhebung differiert.

\subsection{Laienlinguistische Konzepte}

Die 60 Probandinnen und Probanden, die im Ländere ${ }^{n}$-Projekt befragt wurden, metakommunizieren in den Draw-a-map-Tasks zu einer Kleinraumkarte (mit den Kantonen Ob- und Nidwalden in der Kartenmitte und Teilen der Kantone Luzern, Bern, Schwyz und Uri an den Kartenrändern) zwischen 0 und 9 laienlinguistische Konzepte, die fast ausschliesslich den mental konstruierten Raum Ob-/ Nidwalden betreffen (vgl. Abbildung 1). Im Mittel entspricht dies vier Konzepten pro Proband/in (Mittelwert $=3.93$ ) bei einer vergleichsweise homogenen Verteilung (Median $=4)$.

serst sorgfältig und zurückhaltend agiert. Aber schon allein die Tatsache, dass der inhaltliche Fokus bei der Radiosendung Persönlich auf den Biographien der geladenen Gäste liegt, während in einer Dialektbefragung thematisch auf Varietäten, Varietätengebrauch und Einstellungen/Bewertungen von Varietäten fokussiert wird, konstituiert derart unterschiedliche Settings, dass mit unterschiedlicher Quantität und Qualität von anfallenden Metakommunikaten gerechnet werden muss. 


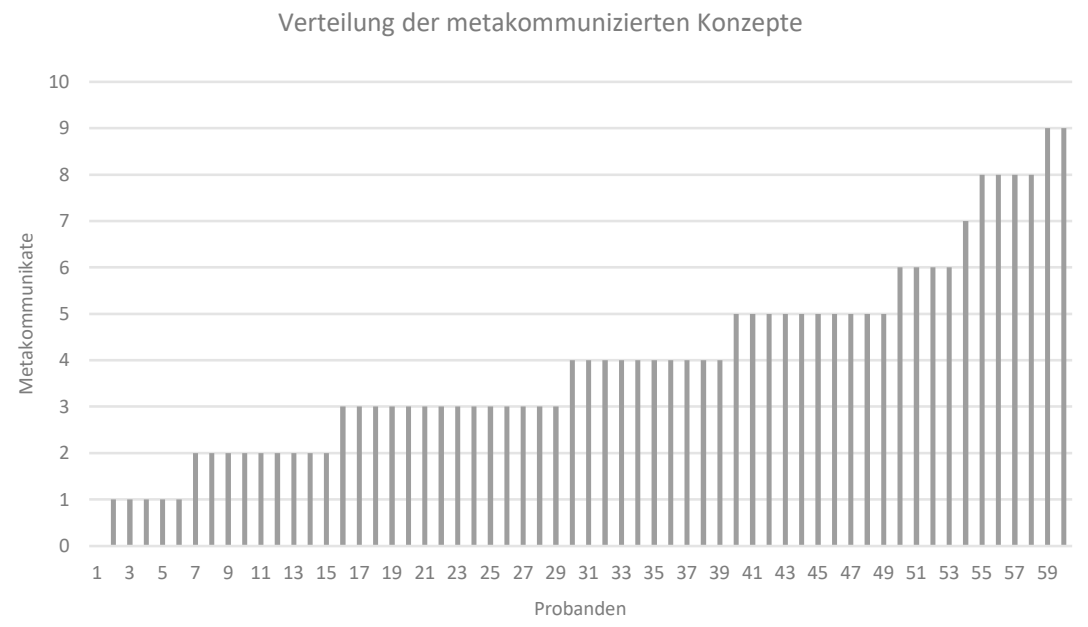

Abb. 1: Übersicht über die Verteilung der von den 60 Probandinnen und Probanden metakommunizierten Konzepte.

Ausgehend von dieser Verteilung wird das metakommunikative Verhalten der untersten wie auch die obersten $10 \%$ der Probandinnen und Probanden exemplarisch diskutiert: Sie verbalisieren 0-1 resp. 8-9 laienlinguistische Konzepte. ${ }^{15}$

Die einzige Probandin im Sample, die kein laienlinguistisches Konzept verbalisiert, ist Probandin PB9 aus dem obwaldnerischen Engelberg. Dies hat in ihrem Fall damit zu tun, dass sie sich weigert, den Draw-a-map-Task zu erledi-

15 Dass maximal nicht mehr Nennungen zusammenkommen, mag erstaunen, hat in erster Linie allerdings mit der Art der Zählung zu tun, die z. B. von den Zählungen von Anders (2010), Stoeckle (2014) und Hundt (2017) abweichen. Und zwar wurde bei der Durchsicht der Daten einerseits auf konkrete Merkmalsnennungen geachtet (hierarchiehöchste Ebene bei Hundt 2017). Zudem wurden die Merkmalsnennungen nicht je einzeln gezählt (Huis ,Haus‘, Muis ,Maus“ = 2 Metakommunikate), sondern es wurde eruiert, welche Entsprechungsklassen bei den Probandinnen und Probanden mental repräsentiert sind. Mit Entsprechungsklasse wird hier der Sachverhalt begrifflich gefasst, dass das Dialektwissen durch systematische Gegenüberstellungen organisiert wird (,wo ich x sage, sagen bestimmte andere y“). Huis ,Haus“ und Muis ,Maus‘ sind aus dieser Optik somit zwei Beispiele resp. zwei Merkmalsnennungen einer einzigen Entsprechungsklasse. Die genannten Lexeme sind nämlich Träger der Klasse $u i-o ̈ i-$ $u u$, die bei der Probandin/beim Probanden offensichtlich mental repräsentiert ist (vgl. für eine detailliertere Begründung dieser Art der Zählung Schiesser 2020, Kap. 12.2). Diese Art der Kategorisierung und Zählung gibt also Aufschluss darüber, welche Entsprechungsklassen sowohl individuell als auch interindividuell repräsentiert sind. 
gen: In der Schweiz - so ihr Argument - werde ohnehin an jedem Ort anders gesprochen, darum könne sie auf der Karte keine Gebiete einzeichnen. Zudem kenne sie sich geographisch nicht gut aus. ${ }^{16}$

Unter den fünf Probandinnen und Probanden, die bloss ein Konzept verbalisieren - Probandin PB1, Proband PB4, Probandin PB35, Proband PB51 und Proband PB55 - ist Proband PB4 aus Emmetten der einzige, der eine eher unspezifische lautliche Angabe macht. In seinen Kartenzeichnungen unterscheidet er die Gebiete Obwalden und Nidwalden voneinander und kommentiert: Je nachdem, „ob jemand das $a$ oder das $o$ oder das $u$ mehr betont ${ }^{\star 17}$, merke man, aus welchem Gebiet er oder sie komme. Diese Äusserung kann als „unspezifische Ahnung“ (Hundt 2017: 127-128) gelten, die in der Wissenshierarchie ganz unten steht.

Von den übrigen vier Probandinnen und Probanden beziehen sich drei in ihrem einzigen Metakommunikat auf das Konzept der lautlichen Entsprechungsklasse $i i-e i-(u i)$, mit der gemeinhin die Gebiete Obwalden, Nidwalden und Engelberg voneinander abgegrenzt werden.$^{18}$ Bei allen drei Befragten findet sich somit sogenanntes „spezifisches Wissen“ im Sinne von „Einzelmerkmalen“ (Hundt 2017: 135-138). Allerdings lässt die Verbalisierung und Kontextualisierung der genannten Einzelmerkmale darauf schliessen, dass bei Probandin PB35, einer Obwaldnerin, die dialektalen Verhältnisse des Untersuchungsraumes am genauesten mit geographischen Arealen verknüpft sind („Neid, neid... Wir sagen nid. Und die Engelberger nuid.“), während Probandin PB1 und Proband PB55 zwar dieselben geographischen Areale unterscheiden, allerdings aber nur dialektale Merkmale zur Beschreibung eines Areals anführen. Proband PB55 zeigt zudem gleichzeitig Strategien der Wissensverbalisierung, die als „allgemeine Dialektcharakterisierungen“ (Hundt 2017: 129-134) gelten können und eine „unspezifische Ahnung“ (Hundt 2017: 128) erkennen lassen („Weisst du, ich kann dir keine Merkmale sagen.“ resp. „Und der Dialekt, der dort gesprochen wird, ist ähnlich wie unserer, aber ein bisschen feiner.“).

16 Da Probandin PB9 die ihr vorgelegten Karten nicht händisch bearbeitet, ergibt sich auch nicht die Möglichkeit, mit ihr über ihre Dialektraumvorstellungen zu sprechen; insofern fehlen allfällige Metakommunikate zum fraglichen Gebiet. Dies bedeutet keineswegs, dass Probandin PB9 nicht doch Dialektmerkmale zu ihrer näheren wie auch weiteren Umgebung hätte nennen können (vgl. „negatives Varietätenwissen“, Hundt 2017: 125); faktisch aber wurde in der Befragung nicht darauf bestanden, dass sie solche Merkmale nennt.

17 Die dialektalen Kommentare aus dem Ländere ${ }^{n}$-Projekt sind jeweils in die Standardsprache übertragen.

18 Dieses Konzept wird auch vom Gesamtsample der Probandinnen und Probanden am häufigsten metakommuniziert (vgl. Schiesser 2017). 
Schliesslich verweist Proband PB51 mit den Dialektmerkmalen, die er nennt, auf die sprachliche Verschiedenheit von $\mathrm{Ob}$ - und Nidwalden: „Der Obwaldner spricht viel mehr mit Hemmli, Zwenzgi und Hentsche, so was, wo ich Häntsche sage. Es ist ein markanter Unterschied zwischen Nid- und Obwalden.“ Proband PB51 nutzt das Konzept der lautlichen Entsprechungsklasse $e-\ddot{a}$, um Unterschiede im Öffnungsgrad von mhd. $e$ vor Nasalverbindungen - die im Untersuchungsgebiet als objektive Daten auch aktuell belegt sind (vgl. Schiesser 2020) - zu thematisieren. Die Art und Weise seiner Thematisierung - v. a. die Gegenüberstellung des Begriffspaars Hentsche vs. Häntsche - ist durchaus präzise, auch wenn PB51 sich dabei auf stereotype Beispiele bezieht. Eine Kollokation, die von den Probandinnen und Probanden nämlich oft genannt wird, ist „Hemmli, Hend und Hentsche“ für Obwalden versus „Hämmli, Händ und Häntsche“ für Nidwalden (,Hemd, Hände und Handschuhe‘). Proband PB51 ist in der Lage, diese Kollokation - für den Alltagsgebrauch als eingängige Trochäen rhythmisiert - zu durchschauen und sie mit der richtigen Entsprechungsklasse in Verbindung zu bringen: Z. B. zeigt das Benennen einer weiteren möglichen Entsprechung Zwenzgi ,Zwanzigrappenstück', dass er tatsächlich über die Entsprechungsklasse verfügt.

Der Blick auf die fünf Befragten, die vergleichsweise wenig Wissen metakommunizieren, zeigt: Alle beziehen sich in ihren Äusserungen auf lautliche Konzepte. Vier der fünf Probanden scheinen zudem in der Lage zu sein, konkrete Merkmale zu nennen. Die Einzelmerkmale, die die vier Probandinnen und Probanden nennen, entsprechen dem objektiven dialektologischen Befund im Gebiet. Das Merkmalswissen, das dabei zum Ausdruck kommt und wegen seiner Spezifizität Ähnlichkeiten mit Expertenwissen aufweist, könnte dabei - wie weiter unten noch eingehender thematisiert wird - (auch) dem Umstand zuzuschreiben sein, dass es hier um einen Abgleich zwischen sehr ähnlichen Dialekten geht.

Verlagern wir den Blick nun auf jene sechs Befragten, die verhältnismässig viel laienlinguistisches Wissen verbalisieren: Proband PB16, Proband PB20, Probandin PB23, Probandin PB27, Proband PB28 und Proband PB41 nehmen in ihren Äusserungen Bezug auf 8-9 Konzepte (vgl. Tabelle 1). Sie sind von der Granularität des Wissens her, das sie metakommunizieren, allesamt in der hierarchiehöchsten, „expertenähnlichen“ Wissensschicht anzusiedeln. Auch ihre Verbalisierungstechniken sind ausgefeilt: Fast sämtliche der sechs Personen metakommunizieren ihre laienlinguistischen Konzepte in einer Weise, die es erlaubt, sie an dialektale Grössen anzubinden. Zudem sind die metakommunizierten Merkmale in der Regel vereinbar mit dem objektiven sprachlichen Befund im Untersuchungsgebiet. Unterschiede gibt es bezüglich der linguistischen 
Beschreibungsebenen, denen die metakommunizierten Konzepte angehören. Hier gliedert sich das Verhältnis zwischen lautlichen, morphologischen und lexikalischen Metakommunikaten je nach Proband/in anders.

Einzig Probandin PB27 und Proband PB41 etwa nennen morphologische Merkmale. Sie führen das regionalspezifische Diminutiv -ili an, das laut SDS (III: Karten 149-154) für den Raum Nidwalden und den Raum Entlebuch belegt ist. ${ }^{19}$ PB27 verweist darauf, dass die Nidwaldner „häufig die Verkleinerungsform Rugili“ bräuchten. Sie selbst sage „Rugeli“. PB41 erklärt in Bezug auf Nidwalden: „Die sagen $d$ Meitili, das ist die Verkleinerungsform ....“. Dass nicht mehr Probandinnen und Probanden - und auch solche nicht, die viel Dialektwissen verbalisieren - morphologische Merkmale metakommunizieren, ist ein Befund, der sich mit dem Gesamtsample des Projektes wie auch mit weiteren Forschungsarbeiten deckt (vgl. z. B. Anders 2010; Stoeckle 2014; zur Nennung einer flexivischen Besonderheit s. unten PB41). Es scheint demnach so zu sein, dass die morphologische Spezifität dialektaler Merkmale den Probandinnen und Probanden nicht oder schwerer ins Bewusstsein rückt resp. im Prozess der Verbalisierung weniger zugänglich ist, wobei ,semantischere' Wortbildungsaffixe sich noch ansprechbarer zeigen als Flexionsaffixe. ${ }^{20}$

Tab. 1: Übersicht über die metakommunizierten Konzepte, sortiert nach linguistischen Beschreibungsebenen.

\begin{tabular}{|c|c|c|c|c|}
\hline Proband/in & Konzepte & Lautung $^{21}$ & Morphologie & Lexik $^{22}$ \\
\hline PB16 & 8 & $\begin{array}{l}i i-e i-(u i), u i-o ̈ i-u u, \\
a i-o i-a u, \text { Länge - } \\
\text { Kürze }\end{array}$ & & $\begin{array}{l}\text { Beggried, heuwä, huere, } \\
\text { meineid }\end{array}$ \\
\hline
\end{tabular}

19 Dass die Probanden PB27 und PB41 mit -ili auf ein Merkmal verweisen, das für den Raum Nidwalden objektiv betrachtet nicht singulär ist, sondern laut SDS auch für den Raum Entlebuch belegt ist, zeigt, dass nur Obwalden und Nidwalden aufeinander bezogen werden, nicht aber weitere in der Nähe liegende Areale. Diese entsprechen für die Probandinnen und Probanden offenbar nicht relevanten Bezugsgrössen.

20 Preston (1996) unterscheidet unterschiedliche Grade der Zugänglichkeit laienlinguistischen Wissens: unavailable (für Laien nicht zugänglich), available (für Laien im Gespräch mit einem Explorator zugänglich), suggestible (durch einen Explorator elizitierbar) und common (Teil alltäglicher laienlinguistischer Diskussionen).

21 Die lautlichen Metakommunikate sind nach der Häufigkeit sortiert, mit der sie im gesamten Sample (60 Probandinnen und Probanden) auftreten: Die Entsprechungsklassen, die am öftesten interindividuell repräsentiert sind, $i i-e i-(u i)$, finden sich oben in der Auflistung.

22 Die lexikalischen Metakommunikate sind alphabetisch geordnet. 


\begin{tabular}{|c|c|c|c|c|}
\hline Proband/in & Konzepte & Lautung $^{21}$ & Morphologie & Lexik $^{22}$ \\
\hline PB20 & 8 & $\begin{array}{l}i i-e i-(u i), u i-o ̈ i-u u, \\
a i-o i-a u, e e-\ddot{o} o ̈, \\
\text { schniie-schneie, buue } \\
\text { - baue, Chääs - Chees }\end{array}$ & & $n u$ \\
\hline PB23 & 9 & io-ue & & $\begin{array}{l}\text { Anke, Beggried, Buechs, } \\
\text { Fazzenetli, Horb, Kalazze, } \\
\text { Parapluie, recke }\end{array}$ \\
\hline PB27 & 8 & $\begin{array}{l}i i-e i-(u i), u i-o ̈ i-u u, \\
i o-u e, a i-o i-a u, u-l\end{array}$ & $i l i-e l i$ & Laiwi, Pfäister \\
\hline PB28 & 8 & $\begin{array}{l}i i-e i-(u i), u i-o ̈ i-u u \text {, } \\
\text { io - ue, häind - hend } \\
\text { usw., } u-l\end{array}$ & & Chneu, eister, häiwe \\
\hline PB41 & 9 & $\begin{array}{l}i i-e i-(u i), u i-o ̈ i-u u, \\
i o-u e, e-\ddot{a}, \text { häind - } \\
\text { hend usw., ee - öö }\end{array}$ & $i l i-e l i$ & Meigi, Tschifeler \\
\hline
\end{tabular}

Alle sechs Probandinnen und Probanden nennen hingegen - im weitesten Sinne - lexikalische Beispiele: Es scheint sich dabei um eine Kategorie von Dialektmerkmalen $\mathrm{zu}$ handeln, die leichter Eingang findet in den laienlinguistischen Diskurs. ${ }^{23}$ Indes kann die regionale Spezifik der genannten lexikalischen Merkmale - anders als bei den morphologischen und lautlichen - aus objektivdialektologischer Perspektive nicht immer bestätigt werden. Partikeln wie huere ,sehr' oder meineid ,sehr (PB16) werden im Untersuchungsgebiet aktuell zwar tatsächlich gebraucht, ihr Gebrauch ist allerdings nicht auf das Untersuchungsgebiet beschränkt. Beim Lexem Anke ,Butter‘ (PB23), handelt es sich um die Dialektvariante eines früher gemeinschweizerdeutschen Lexems, das mehr und mehr von der standarddeutschen Variante Butter verdrängt wird - dies aber nicht nur im Untersuchungsgebiet, auch in anderen Schweizer Dialektgebieten (vgl. Christen, Glaser \& Friedli 2019: 340). Eister ,immer (PB28) ist ein Lexem mit vergleichbarer Charakteristik, kann es doch in einem grossen Deutschschweizer Areal für die letzte Jahrhundertmitte ausgewiesen werden (vgl. SDS VI: Karte 26); heute dürfte es aber zumeist durch immer ersetzt worden sein. Wie

23 In diesem Kontext ist die Salienzdiskussion angesprochen, die danach fragt, warum gewisse sprachliche Merkmale die Aufmerksamkeit der Sprecherinnen und Sprecher eher erlangen als andere, somit eher mental repräsentiert sind und infolgedessen metakommuniziert werden können. Den vorliegenden Überlegungen liegt dabei die Idee der soziolinguistisch bedingten Salienz zu Grunde, die sich von einer physikalisch resp. kognitiv bedingten Salienz unterscheidet (vgl. Auer 2014). 
die objektiven Daten der Befragung zeigen, scheint sich eister im oberen Teil des Kantons Obwalden allerdings noch halten zu können, sodass es sich in den letzten Jahrzehnten zu einem Lokalspezifikum entwickelt haben könnte. Bei Pfäister ,Fenster، (PB27) handelt es sich um eine Lexemvariante, bei der der klitische pluralische Definitartikel $d$ amalgamiert und die Ausdrucksstruktur entsprechend verändert wurde. Auch das Geltungsareal von Pfäister reicht(e) weit über die Innerschweiz hinaus.

Nebst diesen vermeintlich regionalen Lexemen werden zudem solche genannt, die keine durch zahlreiche lexikalische Träger breit abgestützten Entsprechungsklassen aufweisen, und die aufgrund regionaler Aussprache relativ idiosynkratische Wortformen zeigen. Hier zu nennen ist Chneu ,Knie“ (PB28) oder häiwe/heuwä ,heuen‘ (PB16, PB28) ${ }^{24}$ sowie Laiwi ,Lawine‘ (PB27). Bei nu ,noch“ (PB20) kommen keine anderweitig gültigen Lautregeln zum Zuge. Ebenfalls thematisiert werden regionalspezifische Aussprachen von Ortsnamen: Beggried ,Beckenried‘, Buechs ,Buochs‘, Horb ,Horw‘ (PB16, PB23).

Fazzenetli ,Taschentuch' und Kalatze ,Frühstück' (sic!'25) (PB23), die beide auf romanische Wurzeln zurückgehen, sind dagegen Nennungen, die im engeren Sinn als lexikalische Spezifitäten des Unterwaldner Raums gelten können, wobei das Verb kalatze allerdings früher in allen Urschweizer Kantonen gebräuchlich war (vgl. SDS V, Karte 155). ${ }^{26}$ Gültigkeit für den Unterwaldner Raum haben auch die nachbarschaftlichen Neckbezeichnungen Meigi ,Nidwaldner und Tschifeler ,Obwaldner' (PB41), die als Fremd-, nicht jedoch als Eigenbezeichnung gebraucht werden.

Die Probandin PB23, die mit acht Nennungen weitaus am meisten Konzepte benennt, verbalisiert fast ausschliesslich lexikalische Merkmale. In ihren Nennungen spiegelt sich damit die Problematik, die oben beschrieben wurde: Nicht alle der von ihr genannten Merkmale entsprechen regionalspezifischen Lexemen im engeren Sinn, vielmehr hält sie vor allem veralteten Wortschatz für dialektspezifisch. Dies kann immer wieder beobachtet werden und sagt mehr über Dialektstereotype (Dialekte als Sprachformen, die einen alten Zustand

24 Hierbei handelt es sich um Lexeme, die Träger sind von Entsprechungsklassen mit nur wenigen lexikalischen Mitgliedern.

25 Die Nennung des Lexems Kalatze, das den Status eines Substantivs haben soll, macht dessen Ungebräuchlichkeit deutlich: kalatzen lautete nämlich ursprünglich das Verb für ,frühstücken', Kalatz dagegen das Substantiv für ,Frühstück'.

26 Probandin PB32, die mit Abstand am meisten lexikalische Merkmale nennt, reiht hier auch Parapluie ,Regenschirm‘ ein. Zum Nidwaldner Dialekt sagt sie: „Es ist ein relativ breiter Dialekt, der auch noch viel französische Elemente beibehalten hat. Mein Grossvater hat oft vom Parapluie gesprochen und vom Fazzenetli.“ 
bewahren) als über spezifisches Dialektwissen aus. Da Metakommunikate von PB23 - im Vergleich zu den übrigen fünf betrachteten Probandinnen und Probanden - sehr stark auf diese lexikalische Kategorie eingeschränkt zu sein scheinen, wäre den übrigen Probandinnen und Probanden wohl insgesamt ein differenzierteres Dialektwissen zu attestieren.

Am häufigsten werden von den sechs Probandinnen und Probanden Merkmale der lautlichen Beschreibungsebene thematisiert. Ganz prominent metakommuniziert werden dabei die Konzepte der lautlichen Entsprechungsklassen $\ddot{i}-e i-(u i)$ und $u i-\ddot{o} i-u u$, dies häufig mit Rückgriff auf die Strategie des Vergleichs: Proband PB41 erklärt zu Nidwalden: „Die sagen $d$ Meis anstatt $d$ Miis, $d$ Leit anstatt $d$ Liit.“ Probandin PB27 erklärt zu Engelberg: „Die haben das ui noch ganz ausgeprägt: Am fuif vor fuifi.“ Und Proband PB41 erklärt den Unterschied zwischen Uri und Obwalden: „Eigentlich, wenn man es gesamtschweizerisch anschaut, haben sie viel gemeinsam mit uns Obwaldnern. Sie haben ein $\ddot{u}$... Sie haben viel das $\ddot{u}$ drin, wo wir das ui haben.“

Ebenfalls häufig metakommuniziert werden die Konzepte io - ue (PB28 mit Bezug auf Giswil: „Die sagen guet und nicht giot. Die Wörter sagen sie einfach anders als wir.“) sowie $a i-o i-a u$ (PB16 aus Hergiswil: „Wir sagen Baum und nicht Baim."), die auf regionale Spezifika anspielen.

Die übrigen lautlichen Konzepte werden nur von einzelnen Befragten metakommuniziert und widerspiegeln damit nicht unbedingt kollektives, sondern eher individuelles Wissen. Sie beziehen sich auf je andere, z. T. regional stark eingeschränkte lautliche Befunde, die den Probandinnen und Probanden offenbar durch Kontakte in Beruf oder Freizeit bewusst geworden sind. Proband PB41 beispielsweise thematisiert explizit, dass er - als Politiker - besonders darauf achtet, „scheen statt schöön“ zu sagen, um mit der Entrundung von mhd. œ seiner sprachlichen Ingroup zu entsprechen. Weiter verbalisiert PB41 dialektales Spezialwissen zum Ort Lungern (,Die sagen sie häind für ,sie haben', nicht sie hend ... $d$ Biobe. Naturjuzer haben sie schöne ...“), das offenbar seinem Interesse an Volksmusik und Volksbräuchen entspringt.

\subsection{Laienlinguistische Perzepte}

Kontrastierend $\mathrm{zu}$ den besprochenen Daten unter 3.1 sollen - unter Rückgriff auf die These von Hundt (2017: 138), dass die Verbalisierung sprachlichen Wissens kontextabhängig sein kann - folgende Fragen diskutiert werden: Geben vorliegende Daten Hinweise darauf, dass die Befragten, die wenige resp. viele Konzepte metakommunizieren, ebenso wenige resp. viele Perzepte äussern? Und wie steht es um die inhaltliche Beschaffenheit der metakommunizierten 
Grössen: Bestehen bei den einzelnen Probanden qualitative (Teil)Übereinstimmungen zwischen Konzepten und Perzepten? Diese Fragen werden mit Blick auf die „Hörproben-Aufgaben“ diskutiert, von denen hier nur jene in den Blick genommen werden, die als dialektale Repräsentationen des Gebietes von Ob- und Nidwalden gelten können und damit die mentalen Räume $O b$ - und Nidwalden ansprechen, die im Draw-a-map-Task die herausragende Rolle spielen. 

Anzahl metakommunizierter
Anzahl metakommunizierter
Konzepte pro Proband/in
Perzepte pro Proband/in



Abb. 2: Gegenüberstellung der verbalisierten Konzepte. 
Bezüglich der Frage zu den quantitativen Unterschieden erscheinen drei Aspekte aufschlussreich (vgl. Abbildung 2):

1. Die Auswahl der hier in den Blick genommenen Probandinnen und Probanden erfolgt nach dem Ausmass der verbalisierten Konzepte: Jene mit den wenigsten (0-1 Konzepte) wurden jenen mit den meisten Nennungen (8-9 Konzepte) gegenübergestellt. Die quantitativen Verhältnisse bei den genannten Perzepten sind nun insofern nicht spiegelbildlich, als sich hier bei den Befragten das nahezu gesamte quantitative Spektrum zwischen 2 und 14 Metakommunikaten zeigt (dies bei einem Mittelwert und einem Median von 8 Metakommunikaten). Es ist somit davon auszugehen, dass der auditive Stimulus auch bei Probanden mit wenig resp. mit schwer abrufbarem laienlinguistischem Wissen das Abrufen dialektalen Wissens tendenziell begünstigt.

2. Blickt man jedoch auf die Anzahl der Metakommunikate pro Proband und Erhebungskontext, scheint sich die Rangfolge der Probanden nicht wesentlich zu unterscheiden: Jene Befragten, die schon im Draw-a-map-Task viel resp. wenig Wissen verbalisiert haben, verbalisieren auch bei der Hörproben-Aufgabe viel resp. wenig Wissen: Die meisten von ihnen liegen in beiden Tests entweder oberhalb resp. unterhalb des Medians (4 resp. 8 Metakommunikate), d. h. das quantitative Verhältnis zwischen metakommunizierten Konzepten und Perzepten ist bei den meisten Befragten ähnlich.

3. Diese Beobachtung trifft allerdings nicht auf alle Befragten zu: Die Abbildung zeigt nämlich zusätzlich, dass es Probandinnen und Probanden gibt, die beim Draw-a-map-Task nur wenig, bei der Hörproben-Verortung hingegen viel Wissen bekunden. Probandin PB4 etwa verbalisiert lediglich ein Konzept, dagegen aber acht Perzepte und liegt damit im Mittel der befragten Personen. Obwohl ihr die korrekte Verortung der beiden Hörproben nicht ganz gelingt, so zeigt sie doch elaborierte metasprachliche Strategien, die sich in diesem Kontext als erfolgreich herausstellen: Sie setzt bestimmte perzipierte Dialektformen in Vergleich zu eigenen Dialektformen (,D Mueter fällt mir auf, ich würde sagen ds Müeti.“, „Gschnit wäre bei uns gschneit.“) oder aber in Vergleich zur vermuteten Ziel-Varietät (,holt klingt etwas anders“). Insgesamt wird deutlich, dass Probandin PB4 in der Lage ist, perzipierte Dialektmerkmale mental einzuordnen und $\mathrm{zu}$ einem eigenständigen Urteil zu gelangen.

Proband PB51 verhält sich ähnlich: Im Draw-a-map-Task nimmt er auf ein einziges Konzept Bezug, in der Hörproben-Verortung auf zwölf Perzepte, und auch er setzt die metasprachliche Strategie des Vergleichs ein, der sich letztlich die 
Entstehung der hier geltend gemachten Entsprechungsklassen verdankt. Ein Aspekt ist zusätzlich bemerkenswert: So äussert PB51 in der Hälfte der zweiten Hörprobe nämlich, er sei gespannt auf die Realisierung des Wortes „Handschuhe“ ${ }^{\text {27 }}$ - und entscheidet sich, nachdem er das Lexem in der entsprechenden Lautung gehört hat, für die richtige Verortung des Sprechers. Er reagiert demnach nicht erst auf perzipierte Merkmale, sondern er antizipiert bereits, was kommen und ihm bei der Verortung helfen könnte, $d$. h. er verfügt über eine Art von Checkliste mit einzelnen Merkmalen, die die offenbar alltagsrelevante Lokalisierung sicherstellt. Es sind diese mental repräsentierte Dialektmerkmale, die PB51 im Draw-a-map-Task von sich aus nicht verbalisiert hatte.

Ein Proband, der sich gerade umgekehrt verhält, ist PB28. Beim Draw-amap-Task verbalisiert er ausnehmend viele, bei der Hörproben-Verortung hingegen lediglich sieben Merkmale und bleibt damit hinter dem interindividuellen Durchschnitt zurück. Bei der genauen Betrachtung seiner Bearbeitung der Hörproben-Aufgabe fällt allerdings auf, dass er lediglich jene Merkmale metakommuniziert, die ihn in seiner Annahme der korrekten Verortung bestätigen oder widerlegen - was insgesamt zu einer geringen Zahl verbalisierter Merkmale führt. Die schiere Quantität der Metakommunikate allein ist hier demnach nicht aufschlussreich genug: Die Entschlossenheit, mit der PB28 Einzelmerkmale als Erkennungsmerkmale der einen resp. der anderen Sprechergruppe einschätzt, lässt nämlich auf ein fundiertes Dialektwissen schliessen, das sich auch in der korrekten - Verortung der Hörproben spiegelt.

Bezüglich der Frage zu möglichen qualitativen Korrespondenzen zwischen metakommunizierten Konzepten und Perzepten ist Folgendes zu beobachten: Von den Befragten, die lediglich ein laienlinguistisches Konzept metakommunizieren, findet sich dieses geäusserte Konzept bei drei von fünf Befragten auch in den metakommunizierten Perzepten wieder (PB1, PB51, PB55). Probandin PB1 und Proband PB55 etwa thematisieren sowohl beim Draw-a-map-Task als auch bei der Hörproben-Aufgabe die lautliche Entsprechungsklasse $i i-e i-$ (ui). Beachtenswert daran ist, dass bei den Hörproben nur der Trigger ii vorkommt, und nicht etwa sein Pendant ei oder ui. Diesen Umstand kann man als Indiz dafür werten, dass Entsprechungsklassen tatsächlich kognitiv real sind, reicht doch ein Element aus einer Entsprechungsklasse aus, um die ganze Entsprechungsklasse zu aktivieren. Proband PB51 thematisiert im Draw-a-map-Task die lautliche Entsprechungsklasse $e-\ddot{a}$, die in der Hörprobe über die Entsprechun-

27 Dass dieses Lexem sowohl in der Sarner als auch in der Stanser Hörprobe auftauchen wird, ist ihm klar, da er vorher bereits einige Hörproben von Sprechern aus anderen Orten gehört hat. 
gen Häntsche resp. Hentsche in Erscheinung tritt. Aufschlussreich ist hier der bereits oben diskutierte Umstand, dass PB51 - noch bevor Häntsche in einer Hörprobe verbalisiert wurde - metakommuniziert, es werde dieses Wort sein, das ihm bei der Verortung der Hörprobe dienlich sein würde. Diese Aussage ist ebenfalls ein Indiz dafür, dass die Entsprechungsklasse $e-\ddot{a}$ ein solide repräsentiertes mentales Konzept darstellt.

Keine Übereinstimmung zwischen geäusserten Konzepten und verbalisierten Perzepten zeigt sich bei Proband PB4 und Probandin PB35. Proband PB4 der im Draw-a-map-Task keine linguistisch fassbaren Merkmale metakommuniziert - verbalisiert in der Hörproben-Aufgabe dagegen acht Perzepte (s. o.). Ihm scheint der auditive Stimulus bei der Verbalisierung von Dialektmerkmalen zu helfen. Anders ist dies bei Probandin PB35: Sie - die im Draw-a-map-Task lediglich ein Konzept metakommuniziert - verbalisiert in der Hörproben-Aufgabe zwei Perzepte, von denen keines mit dem geäusserten Konzept übereinstimmt. Dieses Konzept - die lautliche Entsprechungsklasse io - ue - scheint sie bei der Hörproben-Aufgabe demnach nicht ohne weiteres aktivieren zu können. Diese Beobachtung untermauert die Einschätzung, wonach es sich hier um eine Person mit vergleichsweise schwachen metakommunikativen Fähigkeiten handelt.

Bei den Befragten, die viele laienlinguistische Konzepte metakommunizieren, findet sich die Mehrheit dieser verbalisierten Grössen auch als metakommunizierte Perzepte. Proband PB16 etwa thematisiert drei der vier lautlichen Konzepte $^{28}$, die er im Draw-a-map-Task nennt, auch in der Hörproben-Aufgabe, zwei davon ausführlich. Bei der ersten Hörprobe etwa formuliert er mit Bezug auf die Entsprechungsklasse $a i-o i-a u$ : „Entweder ist es ein Obwaldner oder ein Nidwaldner. Ich kann es noch nicht definieren, weil er oi oder au noch nie gesagt hat.“ Auch das von ihm verbalisierte Konzept Länge - Kürze taucht bei den Perzepten wieder auf: „Der Obwaldner sagt packt $i$ statt packt ii.“ Ein ähnliches Verhalten lässt sich bei weiteren drei Probanden beobachten, die vier von sechs (PB20) resp. vier von fünf (PB27, PB28) lautliche Konzepte auch bei der Hörprobe thematisieren. Einzig Proband $\mathrm{PB}^{29}{ }^{29}$ verbalisiert nur zwei seiner sechs Konzepte auch als Perzepte, was in seinem Fall wohl in erster Linie damit zu tun hat, dass er bei der Hörproben-Aufgabe nur jene sprachlichen Grössen

28 Gezählt werden nur die lautlichen Konzepte und ausschliesslich jene, die in der „Hörproben-Aufgabe“ potentiell perzipiert werden können.

29 Aus dem Raster fällt auch Probandin PB23: Sie, die im Draw-a-map-Task fast nur lexikalische Grössen nennt, die allerdings in der Hörproben-Aufgabe fehlen. Aber auch die einzige lautliche Entsprechungsklasse, auf die PB23 im Draw-a-map-Task Bezug nimmt (io - ue), nennt sie bei der Hörproben-Aufgabe nicht, obwohl die lautlichen Trigger dafür vorhanden wären. 
thematisiert, die für seine Entscheidungsfindung massgebend sind, und nicht alle, die ihm auffallen (vgl. die Ausführungen oben).

Der Blick auf die Mikroebene des situativ je unterschiedlichen metakommunikativen Verhaltens der untersuchten Probandinnen und Probanden zeigt: Wie von Hundt (2017: 125) vermutet, scheint es so zu sein, dass unterschiedliche methodische Settings qualitativ und quantitativ je unterschiedlich geartetes Laienwissen zu Tage fördern können. Vorliegende Analyse illustriert beispielsweise, dass das Laienwissen beim Draw-a-map-Task - erwartungsgemäss heterogener ausfällt als bei der Hörproben-Verortung, bei der die gegebenen auditiven Stimuli die Wahrnehmung lenken. Der Befund, dass aber (fast) alle Probandinnen und Probanden in der Lage sind, sowohl Konzepte als auch Perzepte zu verbalisieren, macht deutlich, dass mit verschiedenen Befragungsmethoden erfolgreich Laienwissen erhoben werden kann.

Probandinnen und Probanden verfügen über mehr oder weniger Dialektwissen. Bei den Probandinnen und Probanden, die wenig Konzepte verbalisiert haben, gab es bei den geäusserten Perzepten beträchtliche Unterschiede: Gewisse Befragte zeigten auch bezüglich Perzepten wenig repräsentiertes Wissen an, andere hingegen eine beachtliche Menge. Und letztere waren jene, die die von ihnen verbalisierten Konzepte auch als Perzepte thematisierten. Die meisten Probandinnen und Probanden aber, die viele Konzepte verbalisiert haben, thematisieren dieselben Grössen auch als Perzepte. Dies spricht für eine solide mentale Verankerung dieser Grössen.

\section{Schweiztypisches Laienwissen?}

Ausgehend von der Feststellung, dass in der Schweiz Sprache häufig zum alltäglichen Gesprächsgegenstand gemacht wird, wurde die Praxis, Sprache und Dialekt im Alltag zu thematisieren, unter zwei Blickpunkten beleuchtet.

Die Durchsicht von 45 Sendungen des Radio-Talks Persönlich hat hierbei ergeben, dass in einem Drittel dieser Sendungen Sprach- und Dialektverwendung zum Thema gemacht wird, obschon das Sendungsformat keineswegs auf Metakommunikation sprachlichen Alltagswissens hin angelegt ist. Diese Metakommunikate - sie lassen sich inhaltlich vornehmlich den Dimensionen Verständlichkeit, Evaluation und Verortung von Sprache zuordnen - illustrieren unterschiedliche Kommunikationsbedürfnisse der Sprecher in Bezug auf die sie umgebenden Varietäten. Während die Thematisierung der Verständlichkeit und die Evaluation sprachlicher Aspekte gängige Topoi der metasprachlichen Auseinandersetzung darstellen, zeigt sich bei der Thematisierung räumlicher Ver- 
ankerung von Sprache eine Eigenart, die wohl als schweiztypisch gelten kann: Zwar mögen auch andernorts Laien über ein Wissen darüber verfügen, welche Varianten zu einem Ort, einer Region ,gehören' und welche nicht. In Äusserungen, wie sie in den Beispielen (4) oder (8) belegt sind, kommt aber zusätzlich zum Ausdruck, dass den als autochthon geltenden Dialektausprägungen eine besondere Qualität als den ,eigentlich“ erstrebenswerten Dialekten zukommt. Zum allfälligen Faktenwissen gesellt sich eine evaluative Komponente, die ,guten' von ,weniger gutem‘ Dialekt trennt. Inwiefern dieses Alltagswissen, das sich von nicht-wertendem Expertenwissen unterscheidet, im Deutschschweizer Dialekthaushalt insgesamt eine stabilisierende Rolle spielt, muss offen bleiben.

Dass sprachräumliches Wissen bei Schweizer Dialektsprecherinnen und Dialektsprechern nun vergleichsweise differenziert repräsentiert ist, wird unter dem zweiten Blickpunkt deutlich. Hier wurden laienlinguistische Metakommunikate, die im Forschungsprojekt Ländere ${ }^{n}$ erhoben wurden, auf die Fragen hin besehen, welche der 60 befragten Probandinnen und Probanden wie viele und wie geartete Konzepte resp. Perzepte verbalisieren. Bezüglich der Quantität der verbalisierten Grössen zeigt sich bei den Konzepten eine grössere interindividuelle Heterogentität als bei den stimulusgesteuerten Perzepten. Der qualitative Blick auf die Metakommunikate zeigt, dass die geäusserten Konzepte wie auch Perzepte von ihrer Granularität her in der Regel dem Typus „Spezifisches Wissen: Einzelmerkmal“ (Hundt 2017: 127) zugeordnet werden können und sie zudem dem objektiven Befund im Untersuchungsgebiet entsprechen. Die Aufzählung und Benennung von Merkmalen, die zu einer konzeptuellen oder perzipierten Dialektkategorie gehören, entspricht ganz der aristotelischen Wissenschaftstradition, die Dialektkategorien anhand der Kookkurrenz bestimmter dialektaler Merkmale bestimmt. Der Sachverhalt, dass die Laien in der hier präsentierten Studie Konzepte und Perzepte häufig, wenn auch nicht ausschliesslich, expertenähnlich metakommunizieren, ist wohl zu einem guten Teil dem beträchtlichen Ausmass an struktureller und lexikalischer Übereinstimmung zwischen den Dialekten der Befragten und den erfragten Konzepten resp. Perzepten geschuldet. Diese Übereinstimmung lässt die (wenigen) Unterschiede umso deutlicher hervortreten. Allerdings ist ergänzend zu konstatieren, dass die Konzepte, die im Zuge eines Draw-a-map-Tasks mit einer informationsarmen Schweizerkarte bei denselben Befragten erhoben wurden, trotz ebenfalls bestehender Ähnlichkeit der betreffenden Dialekte, andere Wissensschichten anzeigen und vor allem in ,allgemeinen Dialektcharakterisierungen“ (Hundt 2017: 127) bestehen. Die Befragten aus den Kantonen Nid- und Obwalden verfügen vor allem über spezifisches Wissen diese beiden Kantone betreffend. Selbst die geographische Nähe zu Stadt und Kanton Luzern wirkt sich nicht dahingehend 
aus, dass beispielsweise lautliche Entsprechungsklassen gebildet würden, in denen auch die Luzerner Variante als Element fungierte. Es wird überaus deutlich, dass sich der (sprachliche) Relevanzraum aus den Kantonen Obwalden und Nidwalden konstituiert und die sprachlichen Gegebenheiten dieses Areals die prominenten Bezugsgrössen darstellen. Nur vereinzelt werden Bezüge zu angrenzenden Regionen wie dem Berner Oberland, Schwyz oder Uri hergestellt, und dann zumeist von Befragten, die in deren unmittelbarer Nachbarschaft leben. Dass das ili-Diminutivum zu einem (typisch) nidwaldnerischen Merkmal werden kann, macht deutlich, dass die Verhältnisse ,anderswo“ d. h. ausserhalb des Bezugsraums, in der Regel nicht in den Blick genommen werden (müssen) (vgl. Fussnote 18). Selbst PB41, der sogar explizit eine „gesamtschweizerische“ Sicht geltend macht (s. o.), ist auf Ob- und Nidwalden bezogen.

Das expertenähnliche dialektale Laienwissen zu einem bestimmten Raumausschnitt bringen wir ursächlich mit dessen mutmasslicher Alltagsrelevanz in Zusammenhang, ganz im Sinne der eingangs erwähnten Relevanzsysteme, die Umfang und Granularität des Wissens zu einem Wirklichkeitsausschnitt steuern. Die Metakommunikate lesen wir vor diesem Hintergrund als Indizien dafür, dass eine Obwaldnerin - auch heute noch - eher auf Nidwalden bezogen ist als auf Zug oder Zürich, ein Nidwaldner eher auf Obwalden als auf andere Regionen. So mag denn die Schweiz tatsächlich das Land sein, wo die Laien Dialektexperten sind, allerdings sind sie dies zumeist nicht für die ganze Schweiz.

\section{Literaturverzeichnis}

Ammon, Ulrich (1995): Die deutsche Sprache in Deutschland, Österreich und der Schweiz. Das Problem der nationalen Varietäten. Berlin, New York: De Gruyter.

Anders, Christina Ada (2010): Wahrnehmungsdialektologie. Das Obersächsische im Alltagsverständnis von Laien. Berlin, New York: De Gruyter.

Auer, Peter (2014): Anmerkungen zum Salienzbegriff in der Soziolinguistik. Linguistik online (4), 7-20. Online verfügbar unter https://bop.unibe.ch/linguistikonline/article/view/1569 (letzter Zugriff 23.02.2021).

Britain, David (2016): Sedentarism and nomadism in the sociolinguistics of dialect. In Nikolas Coupland (Hrsg.), Sociolinguistics. Theoretical Debates, 217-241. Cambridge: Cambridge University Press.

Bucholtz, Mary (2003): Sociolinguistic nostalgia and the authentication of identity. Journal of Sociolinguistics 7 (3), 398-416.

Christen, Helen (2008): „....wiu mer das vilich nid ir ganze schwiz verschteit“ - Empirische Erkundungen zur sozialen Praxis des polydialektalen Dialogs. Sociolinguistica 22, 24-47. Christen, Helen, Elvira Glaser \& Matthias Friedli (2019): Kleiner Sprachatlas der deutschen Schweiz. 7. Aufl. Frauenfeld: Huber. 
Glaser, Elvira (2014): Wandel und Variation in der schweizerdeutschen Syntax. Taal en Tongval 66 (1), 21-64.

Hoffmeister, Toke (2019): Laien als Experten und Experten als Laien. Zur Problematik eines etablierten Begriffs. Linguistik online 99 (6), 151-174.

Hundt, Markus (2017): Struktur und Komplexität des linguistischen Laienwissens. In Markus Hundt, Nicole Palliwoda \& Saskia Schröder (Hrsg.), Der deutsche Sprachraum aus Sicht linguistischer Laien, 121-159. Berlin, Boston: De Gruyter.

Preston, Dennis (1996): Whaddayaknow? The Modes of Folk Linguistic Awareness. Language Awareness 5 (1), 40-74.

Preston, Dennis R. (2010): Perceptual Dialectology in the 21st Century. In Christina Ada Anders, Markus Hundt \& Alexander Lasch (Hrsg.), Perceptual Dialectology. Neue Wege der Dialektologie, 1-29. Berlin, New York: De Gruyter.

Ruoss, Emanuel \& Juliane Schröter (2020): Schweizerdeutsch. Sprache und Identität 1800 bis heute. Basel: Schwabe.

Schiesser, Alexandra (2017): Authentizität durch Sprache. Soziosymbolisch relevante Merkmale als Fundus stilistischer Variation. In Helen Christen, Peter Gilles \& Christoph Purschke (Hrsg.), Räume, Grenzen, Übergänge. Akten des 3. Kongresses der Internationalen Gesellschaft für Dialektologie des Deutschen (IGDD), 325-346. Stuttgart: Steiner.

Schiesser, Alexandra (2020): Dialekte machen. Konstruktion und Gebrauch arealer Varianten im Kontext sprachraumbezogener Alltagsdiskurse. Berlin, Boston: De Gruyter.

Schmidlin, Regula \& Rita Franceschini (2019): Komplexe Überdachung I: Schweiz. In Joachim Herrgen \& Jürgen Erich Schmidt (Hrsg.), Language and Space. An International Handbook of Linguistic Variation, Vol. 30, 4: Areale Sprachvariation im Deutschen, 1012-1038. Berlin, New York: De Gruyter.

Schnettler, Bernt (2007): Alfred Schütz. In Rainer Schützeichel (Hrsg.), Handbuch Wissenssoziologie und Wissensforschung, 102-117. Konstanz: UVK Verlagsgesellschaft.

Schütz, Alfred \& Thomas Luckmann (2003): Strukturen der Lebenswelt. Konstanz: UVK Verlagsgesellschaft.

Schwarzenbach, Rudolf (1969): Die Stellung der Mundart in der deutschsprachigen Schweiz. Frauenfeld: Huber.

Sprachatlas der deutschen Schweiz (1962-1997). Begründet von Rudolf Hotzenköcherle. Bd. IVIII. Bern: Francke.

Stoeckle, Philipp (2014): Subjektive Dialekträume im alemannischen Dreiländereck. Hildesheim: Olms.

Stoeckle, Philipp \& Christian Schwarz (2019): Ethnodialektale Räume in der Deutschschweiz. In Andreas Nievergelt \& Ludwig Rübekeil (Hrsg.), athe in palice, athe in anderu sumeuuelicheru stedi. Raum und Sprache, 391-408. Heidelberg: Winter.

Tophinke, Doris \& Evelyn Ziegler (2014): Spontane Dialektthematisierung in der Weblogkommunikation: Interaktiv-kontextuelle Einbettung, semantische Topoi und sprachliche Konstruktionen." In Christina Cuonz \& Rebekka Studler (Hrsg.), Sprechen über Sprache, 205242. Tübingen: Stauffenburg.

Trümpy, Hans (1955): Schweizerdeutsche Sprache und Literatur im 17. und 18. Jahrhundert. Basel: Krebs.

Wichter, Sigurd (1995): Vertikalität von Wissen. Zur vergleichenden Untersuchung von Wissensund insbesondere Wortschatzstrukturen bei Experten und Laien. Berlin: De Gruyter. 